\title{
A review of methods for the study of bumble bee movement
}

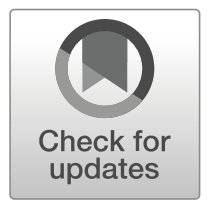

\author{
John M. Mola, Neal M. Williams
}

Graduate Group in Ecology \& Department of Entomology, University of California Davis, One Shields Avenue, Davis, CA 95616, USA

Received 5 January 2019 - Revised 1 May 2019 - Accepted 4 June 2019

\begin{abstract}
Understanding animal movement is critical for conservation planning, habitat management, and ecological study. However, our understanding is often limited by methodological constraints. These limitations can be especially problematic in the study of ecologically and economically important pollinators like bumble bees, where several aspects of their biology limit the feasibility of landscape-scale studies. We review the methods available for the study of bumble bee movement ecology, discussing common limitations and tradeoffs among several frequent data sources. We provide recommendations on appropriate use for different life stages and castes, emphasizing where recent methodological advances can help reveal key components of understudied parts of the bumble bee life cycle such as queen movement and dispersal. We emphasize that there is no one correct method and encourage researchers planning studies to carefully consider the data requirements to best address questions of interest.
\end{abstract}

\section{foraging / dispersal / mark-recapture / radio-tracking / sibship assignment}

\section{INTRODUCTION}

Understanding organismal movement is critical for choosing the appropriate spatial scale over which to consider animal ecology, behavior, or conservation (Hanski 1998, Kremen et al. 2007, Nathan et al. 2008, Allen and Singh 2016). However, our ability to characterize the patterns and estimate the scale of movement is often limited by methodological constraints that make individuals difficult to track and result in inferences made using low-resolution data (Ims and Yoccoz 1997, Cooke et al. 2004, Darvill et al. 2004, Wikelski et al. 2007, Holyoak et al. 2008, Tomkiewicz et al. 2010). Movement is especially challenging to

Electronic supplementary material The online version of this article (https://doi.org/10.1007/s13592-019-00662-3) contains supplementary material, which is available to authorized users.

Corresponding author: J. Mola, jmmola@ucdavis.edu

Manuscript editor: James Nieh quantify for small and fast-moving insects for which many of the methods used on large organisms are not feasible. However, recent improvements in radio-tracking technology (Kissling et al. 2014) and genetic and genomic methods (Andrews et al. 2016), as well as new analytical frameworks (Ovaskainen et al. 2008, Pope and Jha 2017), allow us to better quantify insect movement, with important implications for their application to conservation challenges (Allen and Singh 2016). With these new opportunities comes the need to carefully choose between methods which are differentially suited to examining various types of movement and to consider their associated biases and underlying assumptions, which may influence our downstream inferences.

Bumble bees (Apidae; Bombus spp.) are a particularly important taxon in which to investigate general principles of movement and pollinator ecology. Their eusocial life cycle, involving solitary and social stages with division of labor between reproductive and non-reproducing individuals, combined with a central place foraging 
habit, means that different types of movement (e.g., foraging, dispersal, mate location, habitat selection, and nest searching) contribute to individual, population, and community processes at different scales. The caste and stage-structured life history of bumble bees also allow these different types of movement to be studied and interpreted relatively independently from each other. For example, bumble bee foraging tasks are carried out mostly by non-reproducing workers whose primary motivation is to collect resources for the colony's brood. This compartmentalization of foraging movement from mate location, nest location, and dispersal has allowed for simpler interpretation of its motivations, behaviors, and fitness consequences (Heinrich 1979). Furthermore, when these movement phases are not exclusive, such as when foundress queens may seek nests and forage simultaneously, it allows for a comparison among castes that may help us to understand the impacts of different motivations on resulting movements.

Bumble bee movement is also particularly relevant for pollinator conservation programs because they are highly mobile generalist foragers that access resources from diverse habitats and as such are likely a robust proxy for assessing human impacts on bees generally (Kremen et al. 2002, 2007, Williams et al. 2010). As some of the best documented and easily identifiable bee taxa, their use as indicator species potentially allows for the interpretation of patterns in overall bee declines, with bumble bee species showing clear sensitivity to landscape changes and habitat fragmentation (Rathcke and Jules 1993, Williams et al. 2010, 2011). Bumble bees are also a dominant native pollinator for many crops and wild plants (Free 1993, Klein et al. 2007, Ollerton et al. 2011). Given their importance in a variety of contexts, the reported declines of bumble bee populations in Europe and North America are especially alarming (Goulson et al. 2008, Grixti et al. 2009, Williams and Osborne 2009, Cameron et al. 2011, Jacobson et al. 2018). The loss of suitable forage (Carvell et al. 2006), habitat fragmentation (Darvill et al. 2010), climate-induced range contractions (Kerr et al. 2015), agrochemicals (Rundlöf et al. 2015), and introduced pests and parasites (Plischuk et al. 2009, Li et al. 2012, Arbetman et al. 2013,
Cameron et al. 2016, Aizen et al. 2019) have all been implicated in the recent decline of bumble bee populations (reviewed in Goulson et al. 2008, 2015). Each of these threats occurs within an explicit spatial context, but often, data are lacking on the relevant movement processes that inform models, conservation plans, or experimental designs. For example, a recent study on the potential for range contractions in North American bumble bees under different climate scenarios made substantial assumptions about dispersal distances since no estimates are available for North American species (Sirois-Delisle and Kerr 2018). The study finds starkly contrasting range losses using different dispersal estimates, highlighting the need for more and better estimates of bumble bee dispersal. With a clearer understanding of bumble bee movement and the quantitative data that underlie it, such as those available for larger organisms (reviewed in Nichols and Kaiser 1999, Wikelski et al. 2007, Tomkiewicz et al. 2010, Long and Nelson 2013, Jønsson et al. 2016), the conservation of bumble bee populations and their pollination services can be approached with a more holistic understanding.

At least three key aspects of bumble bee biology limit the ability of researchers to reliably study large-scale foraging or dispersal, which highlights the need for a critical assessment of the available tracking methods. First, bumble bee nests are notoriously difficult to locate, requiring brute-force search efforts which vary widely in success (Harder 1986, Goulson 2009, O'Connor et al. 2012, O’Connor 2013). Second, even with a known colony location, individuals are difficult to detect across large areas, and search effort is rarely commensurate with greater effort required at increasing distance from the colony (Dramstad 1996, Walther-Hellwig and Frankl 2000). Third, workers exhibit strong site fidelity across foraging bouts (Manning 1956, Thomson 1982, 1996, Comba 1999, Cartar 2004, Ogilvie and Thomson 2016), so markrecapture experiments conducted at flowers are often biased towards the redetection of faithful individuals at the same location, resulting in severe underestimates in the scale of movement. Given these limitations, indirect methods of 
tracking bumble bee movement independent of known nest locations have been successfully used (e.g., genetic mark-recapture; see below and Table I), with a set of acknowledged assumptions. Each of these methods may yield idiosyncratic results due to methodological rather than biological differences, and understanding their various biases and limitations is important for interpreting the data they generate.

We provide a critical assessment of methods used in the study of bumble bee movement, linking each method to key questions for which they are especially well suited. We specifically focus on experimental or observational methods, but there are other contexts where museum specimens or population genetic data can also provide key insights into bumble bee movement ecology. A few studies have also capitalized on exotic species introductions and their rates of range expansion to assess intergenerational dispersal (e.g., Morales et al. 2013, Schmid-Hempel et al. 2014). We do not outline these data sources within our review of methods as this information is not normally obtained within a given study to directly observe movement (i.e., researchers are unlikely to introduce species to a non-native range just to study dispersal). Additionally, although captive colonies have given us great insight into finescale foraging behaviors over a few meters, such as floral choice and learning, in contained arena small arrays and patches (e.g., Cameron 1981, Cnaani et al. 2006, Raine and Chittka 2007, 2008, Leonard et al. 2011, Leonard and Papaj 2011), our ability to study movement at the landscape-scale has generally lagged. Therefore, the methods outlined below focus on approaches to investigate field-scale processes of foraging, dispersal, and movement patterns.

For each method, we consider the aspect(s) of movement each technique addresses and summarize the advantages, limitations, appropriate use, and interpretation of the data yielded (see Table I for a summary). Our review will help identify priorities for these new tools, guide researchers through the selection of appropriate methods, and allow readers to more carefully interpret results of insect movement studies with an informed understanding of their underlying methodological constraints.

\section{METHODS IN BUMBLE BEE MOVEMENT ECOLOGY}

Most bumble bee species live in annual colonies with a distinct solitary queen phase, followed by an extended social phase. A colony is founded by a queen that mated the previous year and survived overwintering. This emerging foundress queen disperses from her overwintering site to locate a nesting site. The foundress forages as a solitary individual, building a brood ball composed of pollen and nectar to feed her first cohort of daughter-workers. Once these workers eclose as adults, they assume in-nest and foraging tasks, soon taking over all foraging movements. A reproductively successful colony switches from the production of successive worker cohorts to the production of sexual castes: new queens (gynes) and males. Gynes and males leave the colony to find mates. The mated gynes seek overwintering sites where they remain in diapause until the following spring. The original foundress queen, her daughter-workers, and males all die by the end of the season.

\subsection{Traditional mark-recapture}

The least technical and least expensive method of estimating foraging range is to mark individuals with paint or tags and recapture them away from their nest, typically at flower patches. These measurements provide a distribution of foraging distances from the point of marking. Mean or maximum expected foraging distances can also be estimated assuming some distance-based movement decay function fit to the observed data (Haskell et al. 2002). As such, the method provides measures of displacement but does not reveal flight routes (but see a potential approach used in butterflies where the movement direction depended on the landscape structure; e.g., Sutcliffe and Thomas 1996). However, the displacements can reveal the influence of external factors on movement even if insights into other components of movement such as navigation capacity or internal motivation are unlikely (Nathan et al. 2008).

The primary limitation with traditional markrecapture is the substantial search effort required 
J. M. Mola, N. M. Williams

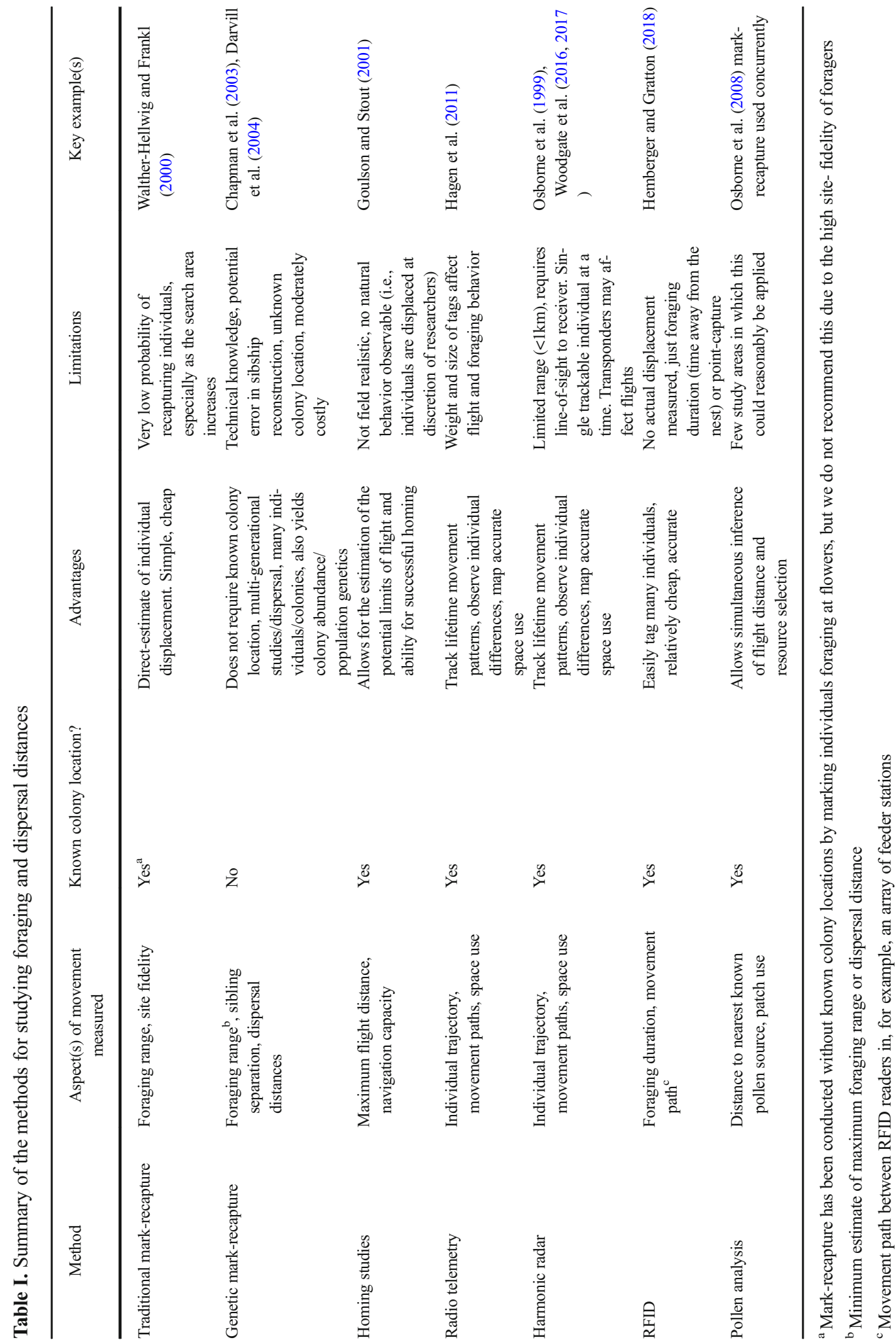


given the low probability of recapturing individuals. Moreover, the effort required to relocate marked individuals increases with the square of the distance from the nest (Walther-Hellwig and Frankl 2000, Darvill et al. 2004), such that robust estimates of longer distance movements become very difficult to obtain. Furthermore, the method requires known nest locations, and sufficient numbers of wild nests are often difficult to locate (Suzuki et al. 2007, O'Connor et al. 2012, O'Connor 2013). This limitation of unknown nest locations can be partially addressed by deploying nests of reared or commercially available colonies. However, this strategy comes with its own limitations in terms of species availability, potential complications of using domesticated species of limited genetic lineages (Gosterit and Baskar 2016), and artificial placement of nests that may not reflect natural locations.

A few studies have marked and recaptured individuals at flowers rather than marking at the nest and used these data to infer estimated foraging range or propensity to move across barriers (Bowers 1985, Bhattacharya et al. 2003, Elliott 2009). This approach is useful for assessing flower-to-flower movements. However, when attempting to estimate landscape-scale foraging distance, such estimates of movement from captures of individuals marked at flowers should be interpreted cautiously because the displacements measured are estimates of the movement away from a previous resource patch and not a movement from a central nest. The high site fidelity of foraging bumble bees (Manning 1956, Thomson 1982, 1996, Comba 1999, Cartar 2004, Ogilvie and Thomson 2016) means that individuals are more likely to be recaptured at the same or nearby patches, even when foragers may have traveled from a distantly located nest. As such, this patch to patch approach underestimates the foraging range. We suggest excluding repeated captures at the same location. Such exclusion is similar to how others have removed very small separation distances $(<50 \mathrm{~m})$ between siblings from genetic mark-recapture analyses (see below; e.g., Carvell et al. 2012).

Despite the landscape-scale limitations of traditional mark-recapture for bumble bees, it still provides an inexpensive and low-tech means to estimate foraging range or space use. Furthermore, mass-marking techniques using powdered dyes (Martin et al. 2006) or protein markers (Hagler et al. 2014, Boyle et al. 2018) at known colony locations can enable substantial sample sizes of marked individuals, with appropriate adjustments made to foraging kernels to account for decreased sampling effort per unit area at more distant recapture locations (e.g., Sivakoff et al. 2012). Recent technological innovation such as protein trapping at the nest may further increase the utility of the approach (R. Isaacs, personal communication). Overall, its application is best suited to situations where colony locations are known and substantial search effort of the surrounding area can be achieved.

\subsection{Genetic mark-recapture (sibship assignment)}

Molecular techniques have been widely used to estimate foraging range and form the basis for most recent studies of field-scale bumble bee movement. The method provides a type of genetic mark-recapture where the reobserved units are putative siblings rather than repeated captures of an individual (originally described by both Chapman et al. 2003 and Darvill et al. 2004, see Supplemental S1 for a full list of studies using similar techniques). At its essential level, freeforaging individuals are collected, genotyped, and assigned to sibling groups (colonies). With known locations of siblings across a study area, basic measurements of a colony's space use can be estimated including sibling separation distance, minimum estimate of maximum foraging range, and number of colonies foraging within a patch. To date, the method has been used primarily to study worker-worker separation distances, but it is also possible to investigate other combinations including worker-male (e.g., Kraus et al. 2009), worker-queen (Lepais et al. 2010, Carvell et al. 2017), and queen-queen distances.

Although there are important assumptions to consider in the interpretation of genetic markrecapture data (described below), it offers several advantages over traditional mark-recapture methods. First, the method does not require known nest locations. Instead, calculations of 
movement are based on the separation distances of free-foraging siblings. Second, although only a few individuals are caught from any given colony, usually many such sibling groups are detected within the collections, allowing for many estimates of separation distance and foraging range. This is an advantage over traditional markrecapture where few marked individuals are ever reobserved. Third, siblings foraging independently can be separated by great distances, even if each individual returns to the same site repeatedly. As such, the complication of individual site fidelity persistent in traditional mark-recapture data is reduced because the "recaptures" occur among siblings. Fourth, genetic mark-recapture is currently the only feasible method for estimating queen dispersal between years. Workers and gynes produced within a colony are full siblings; thus, displacements measured between a foundress and any of her worker-siblings from the previous year can be attributed to dispersal movement. As with foraging range estimates, the accuracy of this method is limited by how well colony location is estimated in year one, as well as by capture rates of related workers and foundresses. Because between-year sibship assignment provides a direct measure of displacement of a colony across years, the method offers a promising avenue for future research on small-scale movements, survivorship, or natal site fidelity within different environments (e.g., Carvell et al. 2017). Lastly, the method provides other data for understanding bumble bee ecology (e.g., colony abundance, colonylevel floral choice, population size estimates, and population genetics metrics). Overall, genetic mark-recapture yields a relatively large dataset without the need for prior information about colony locations, but there are limitations and biases to consider in analysis.

The key limitation in studies using sibship assignment is the lack of known colony locations; however, with proper contextualizing of this unknown, its formalized method for estimating a colony location makes it an especially valuable approach for a range of applications. In all studies of sibship assignment, estimates of foraging range represent minimum distances (hence "minimum estimate of maximum foraging range" being the most commonly reported measurement) because in the absence of other information, the centroid between siblings is assumed to represent the colony's location. Of course, this is likely inaccurate because foragers may concentrate unevenly in space and because few individuals are represented from each sampled colony (Pope and Jha 2017). Although colony location can only be crudely estimated, the separation of individuals can nonetheless help quantify relative displacement (i.e., various distance estimates) and landscape resistance. Furthermore, repeated measurements of sibling separation distance among many sibling groups yield a robust estimate of the relative foraging distance, even if each individual estimate is crude. In practice, if siblings are captured across a potential landscape barrier (e.g., a forest and a mountain pass), at least one of the individuals must have crossed through or around the barrier to explain the displacement between the pair, even though the exact colony location is unknown. Sibling measurements therefore offer a means to compare among species, landscapes, and other ecological factors.

Another source of potential error in estimates of movement is the assignment of individuals to maternal sibships (colonies) itself. Some authors have speculated that error in assignment may help explain some peculiarities in their results (e.g., Lepais et al. 2010, Rao and Strange 2012). Although programs used to reconstruct pedigree, such as COLONY (Wang 2004), are fairly robust to genotyping error, some relationships can be difficult to infer, and different runs or marker sets can yield slightly different results. These differences introduce error into estimates, and researchers should report results across the range of possible pedigrees or include uncertainty in pedigrees directly into downstream analysis (Hadfield et al. 2006). Like the issue of bias from assignment error, vagaries of genetic diversity within populations may also affect estimates based on genetics. For example, a highly inbred population will produce underestimates in the colony number and likely overestimates of movement distance because of false assignment of non-colony mates to the same sibling group. However, with the use of highthroughput sequencing and thousands of informative markers, the risk of error in estimates can be reduced substantially. 
In most studies to date, colony location was not estimated and instead foraging range was inferred only from the separation distance between siblings. Using fine-scale geographic information, it is also possible to assign estimated colony locations to habitat features that are more likely to be suitable nesting substrate (Redhead et al. 2016, Carvell et al. 2017). The accuracy of these estimations remains unclear, and efforts to compare the results of modeled colony locations against known locations of captive colonies remain to be done. Others have instead modeled colony locations across likelihood surfaces using spatially explicit modeling approaches, simultaneously, rather than sequentially, estimating colony location and foraging distances (Pope and Jha 2017, 2018). Despite the numerous studies using sibship assignment, much remains to be explored about how different study designs, levels of sampling effort, analytical frameworks, and supporting data (e.g., known colony locations) can inform the final interpretation of results.

Overall, estimates obtained from sibship assignment qualitatively match results of similar studies using other methods (Figure 1), so appear to be an effective means of collecting many estimates of movement distances without the need for known colony locations or extensive recapture effort. Genetic mark-recapture is likely most useful for relative estimation of foraging range when comparing across species, landscapes, or other ecological contexts. The further extension to estimation of dispersal distances makes it a particularly promising avenue for future research.

\subsection{Homing studies}

Animal homing has been studied extensively to understand movement. Typically, research focuses on the ability of individuals to return to their nests, dens, or regular feeding territories after being experimentally displaced across a range of distances (Fabre 1918, Papi 2012). Homing studies with bumble bees can provide useful estimates of maximum flight distance by displacing individuals at some distance from known colonies and determining return probability and efficiency (Goulson and Stout 2001, Greenleaf et al. 2007). Like studies that mark bees at the nest and recapture them on flowers, these studies provide potential flight distances, but they are less suited for understanding natural space use by individuals or colonies because bees are released at locations they do not choose. As such, return probabilities are strongly affected by navigation capacity and prior experience within potentially novel areas. This unique dimension of experimental displacement can be viewed as a limitation or as an advantage if specifically leveraged to study the influence of external factors and navigation capacity on homing success. Homing studies to date suggest a strong ability to orient (for an example with honeybees; Southwick and Buchmann 1995) and return to a known location from great distances (Goulson and Stout 2001, Rao et al. 2019).

A key advantage of homing studies is that return distances can reveal maximum values, whereas other methods are prone to underestimating foraging range. For instance, in mark-recapture studies, the higher likelihood of recapturing individuals closer to the marking location reduces the final estimates of foraging. Similarly, harmonic radar (discussed below) is limited by the radar's trackable range and likely to miss trips that are more distant. As such, homing can serve as a powerful tool in detecting the maximum foraging potential of bumble bee species. Although only one known study exists using homing methods with bumble bees returning to their colonies (Goulson and Stout 2001), its extensive use with other bee species (see references within Greenleaf et al. 2007) has demonstrated clear connections to behavioral and navigation capacities of bee flight orientation and route selection (Rau 1929, Rossel 1993, Southwick and Buchmann 1995, Collett 1996, Wolf et al. 2014). The method is most practically suited to the use of colonies with known locations, allowing the displacement of hundreds of individuals and ensuring a high detection probability of any returning bees. However, by leveraging the high site fidelity observed in bumble bees, homing ability and flight distances can be estimated by displacing wild foragers. A recent study artificially displaced wild foraging $B$. vosnesenskii from a small flowering patch of Spirea japonica albiflora and found return distances of up to $16 \mathrm{~km}$ and an influence of prevailing wind direction and land cover type on the return times of foragers (Rao et al. 2019). 


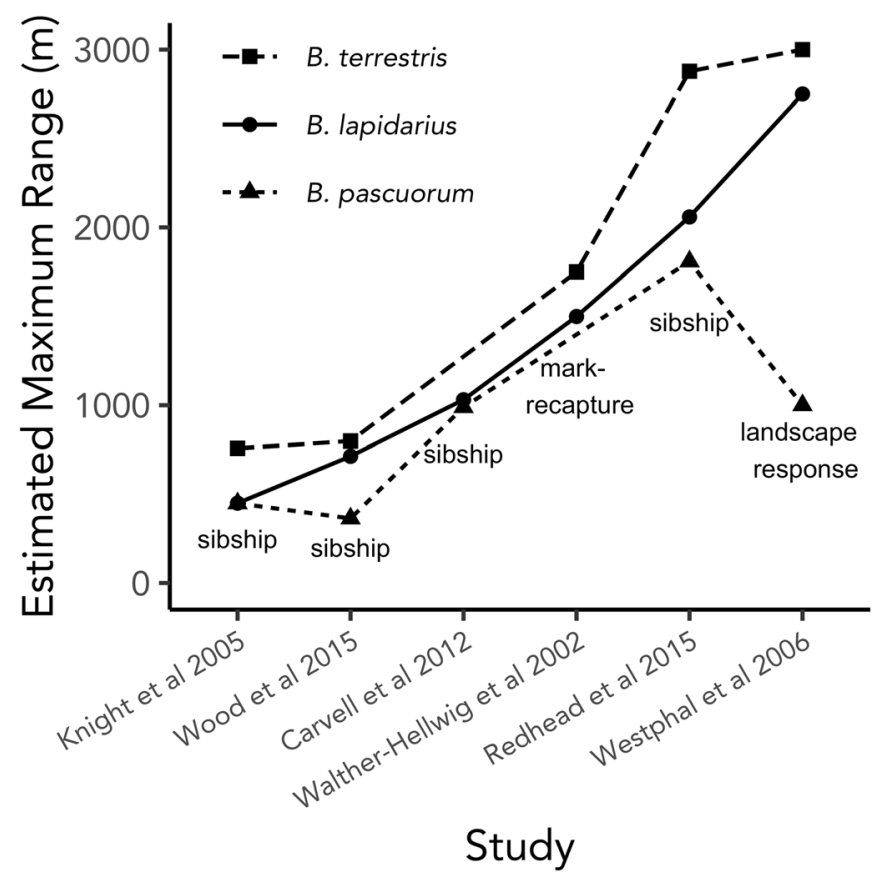

Figure 1 Commonly studied species and their relative foraging range across studies. We used the most commonly studied research species (minimum three studies) and only papers where at least two of those species were studied simultaneously. This resulted in a comparison of B. lapidarius, B. pascuorum, and B. terrestris among five studies. Clear differences exist between species in their estimated foraging range, with $B$. terrestris the longest ranged, B. lapidarius intermediate, and B. pascuorum the shortest. Despite variation in the absolute estimate among studies, the rank order remains consistent allowing clear patterns in species differences to emerge. Labels below points indicate study methodology.

Homing studies may be especially useful under experimental conditions such as understanding how pesticide exposure, landscape context, nutritional status, or other factors affect return speed or frequency across a range of release distances. Homing studies have also been used in combination with harmonic radar to track the return and search patterns of individual honey bees (Wolf et al. 2014) and could be readily applied to studies of bumble bees. Researchers using homing methods should carefully consider whether their results are due to physical limitations (motion capacity of flight) or cognitive limitations associated with navigation capacity and an ability to return from novel or unfamiliar locations. By complementing homing studies with related feeder experiments (e.g., van Nieuwstadt and Iraheta 1996), where foragers are trained to a nectar source that is moved to increasing distances from the nest, cognitive and physical limitations can be partitioned. Although homing studies have yet to be widely utilized for studies of bumble bee movement, their clear interpretation offers a promising means to begin studies of underrepresented species in the literature and estimate their maximum flight range or navigation capacity under various release contexts.

\subsection{Radio telemetry and harmonic radar}

Radio telemetry ("active tagging" with batterypowered tags) and harmonic radar ("passive tagging" with unpowered transponders) have been used to answer questions of space use, flight path optimization, foraging distance, and lifelong movement path in bumble bees (Osborne et al. 1999, Hagen et al. 2011, Lihoreau et al. 2012, Woodgate et al. 2016, 2017; for a general 
review, see Kissling et al. 2014). In both tracking methods, researchers affix a small transponder ("tag") to individuals and using radar stations track their locations over time within some observable radius. These tracking methods differ from all others in that they reveal detailed flight paths and movement behavior with a fine spatiotemporal resolution over potentially thousands of coordinates (as opposed to methods where typically only a few spatial locations are ever known). Within present technical limitations, these methods offer a means to examine all components of the movement ecology paradigm (Nathan et al. 2008). Although the two tagging methods differ in some of their advantages and limitations (distinguished below; reviewed by Kissling et al. 2014), both tracking methods provide a unique way to quantify individual movement paths within the radar's trackable range and as such are worth considering together.

The primary advantage of radar tracking methods over all other methods is the ability to continually track individuals through multidestination or even lifetime movement paths. Tracking allows for estimates of flight distance, duration, and behavioral patterns in movement decisions and can be applied readily to queens, workers, and males across a variety of contexts. Rendering the lifetime movement paths of individual foragers has revealed the ontogeny and constancy in foraging routes and foraging locations (Osborne et al. 1999, Lihoreau et al. 2012). It also has allowed understanding of how habitat quality and other factors may influence movement patterns of foragers from the same colonies over time (Woodgate et al. 2016, 2017) and how individuals differ in movement behavior (Woodgate et al. 2016). Recently, the use of harmonic radar revealed insights into previously unexamined movement behaviors of queens shortly after emergence from overwintering typified by periods of rest and random dispersal (Makinson et al. 2019).

In both tracking methods, the primary limitation is the weight and size of the tags, with a tradeoff between tag weight and power. For radio telemetry, this problem is most acute. The battery required to power the active tags can cause them to weigh substantially more than the passive tags used for harmonic radar (200-1000mg for active tags vs. 1-20 mg for passive; Kissling et al. 2014). The heavy weight of the active tags appears to affect foraging behavior, including causing individuals to forage more slowly and take rest periods that exceed $45 \mathrm{~min}$ (Hagen et al. 2011). Due to these weight concerns, harmonic radar has been favored in the tracking of bumble bees to date (though see Hagen et al. 2011; additionally, see Pasquet et al. 2008, Wikelski et al. 2010 for examples with large-bodied carpenter and orchid bees). Harmonic radar is limited by several issues that make it likely active tagging methods will come to be favored as the weight of the batteries and tags declines. First, harmonic radar is limited to use in relatively simple landscapes. There must be a consistent line-of-sight between the radar dish and the tagged bee, restricting studies to areas that are flat, open, free of barriers, and topographically uniform. With radio tagging, the signal strength of radio tags may be reduced across barriers, but detection is still possible. Second, with passive tags, only one individual can be tracked at a time, although multiple individuals may be tracked either successively or with unknown individual locations (e.g., Makinson et al. 2019). Active tags have a unique signal, so concurrent tracking of several individuals is possible. Third, the trackable range of ground-based harmonic radar is limited to less than $1 \mathrm{~km}$. When using active tags, researchers can use mobile receivers and even aerial surveys to track over longer distances (e.g., Hagen et al. 2011; see White and Garrott 2012 for a review of aerial tracking in large animals). Lastly, despite their smaller relative size, the passive tags may still affect movement by changing flight and foraging behavior (Switzer and Combes 2016), although several studies with bumble bees and honey bees claim there is little influence on the foraging behavior (e.g., Osborne et al. 1999, Capaldi et al. 2000, Woodgate et al. 2016).

Despite the present technical limitations of radar tracking methods, their use has already proven fruitful, and several studies of honey bee foraging demonstrate further applications (e.g., Capaldi et al. 2000, Menzel et al. 2005, Riley et al. 2005, Reynolds et al. 2007, Wolf et al. 2014). Undoubtedly, as the size and weight of tags decline, a shift towards active tagging methods will provide a 
wealth of data and allow detailed studies of bumble bee movement and a revisiting of previous assumptions, just as it has done for studies of other organisms (Kissling et al. 2014).

\subsection{Radio-frequency identification (RFID) and optical tags}

RFID uses smaller tags than those for radar, but tags must be read by readers within a subcentimeter range (Reynolds and Riley 2002, Silcox et al. 2011, Nunes-Silva et al. 2018), making it infeasible for continual long-range detection like radar tracking. It has most successfully been used to follow workers entering and leaving captive nests or feeders (Gill et al. 2012, Hemberger and Gratton 2018, Malfi et al. 2018) but not for direct observations across a large landscape array. For example, it can be used to measure time away from a colony for many individuals, yielding an estimate of the time spent foraging and a proxy for landscape-scale foraging effort (Hemberger and Gratton 2018; for an example without RFID, see Westphal et al., 2006) and colony-wide activity patterns and potential task allocation (Kerr et al. 2019). Like RFID technology, optical tags with QR codes can be used to detect and track individuals within a photographable distance (Crall et al. 2015). Although image storage and processing require significant computing power, these tags are even lighter than RFID tags and are detectable from a greater range. They also have the advantage of continuous monitoring using video recording (Crall et al. 2018). Overall, RFID and optical tagging are of limited use for landscape-scale studies hoping to directly observe movement, but their ability to indirectly reveal various landscape-level factors affecting the frequency and duration of foraging trips in relation to colony location and floral resource structure makes it a promising avenue for future application.

\subsection{Pollen analyses}

Analysis of pollen from returning workers provides an indirect way to track bumble bee worker movements where the location of unique pollen sources is controlled for and/or discretely known (Osborne et al. 2008). Although the primary use of pollen analysis will be for understanding the composition of plant species foragers collect from (Jha et al. 2013), when used in combination with mapping of floral resources, the results can yield estimates of the minimum distance to the nearest known source of that pollen species. New molecular techniques for pollen DNA barcoding and quantification may allow the method to be employed more widely, requiring less expertise in visual pollen identification, and potentially at a finer resolution (Keller et al. 2015, Bell et al. 2016, 2017, McFrederick and Rehan 2016). The use of pollen analysis is likely to be especially valuable in combination with other approaches like mark-recapture where researchers could observe visually distinct returned pollen in real time and use that to inform their search efforts.

\section{COMMON LIMITATIONS AND COMPARISON OF METHODS}

In our review, we find several common methodological limitations. All methods we describe, except for homing studies, tend to underestimate movement distances because of detection biases or omissions at larger distances. Genetic markrecapture and traditional mark-recapture provide the minimum estimate of maximum foraging range, harmonic radar is limited to a radius of detection less than known foraging distances, and pollen analyses only reveal what species was collected and assume the nearest patch as most probable. Studies examining displacement measures (i.e., foraging range, dispersal distance, and sibling separation) further bias towards "nearness" or shorter distances due to the concentration of sampling effort at smaller radii. To avoid it, effort would need to increase proportional with the increase in size of the sampling area. Because this quickly becomes impractical, it can produce a systematic underestimate of foraging range and a bias towards recaptures close to the area of marking. In early studies, this led to the erroneous conclusion that bumble bees forage over short distances (well criticized by Dramstad 1996). Some authors have corrected for "transect end effects" or reduced effort across a range of distances to calculate relative foraging kernels (e.g., Darvill et al. 2004, Jha and Kremen 2013), but 
these corrections are not always made. Simple corrections for effort across annuli of increasing size are also possible and have been implemented for other insects (Sivakoff et al. 2012). These areaeffort corrections will increase the weight of distant recaptures and down-weight nearer recaptures. Combined with area-estimates informed by habitat mapping, our understanding of the commonality of long-distance foraging by bumble bees can be clarified.

Nevertheless, we may view movement as less flexible and dynamic than it is. Given that economic models of foraging suggest that foraging distances exceeding several kilometers can still be profitable (Dukas and Edelstein-Keshet 1998, Cresswell et al. 2000), these constraints on our tracking methods are troubling. We know from studies of the range-expansion rate that queen dispersal distances may exceed tens of kilometers per year (Kadoya and Washitani 2010, Morales et al. 2013, Schmid-Hempel et al. 2014 p. 20), but it is highly unlikely that a reliable search effort could occur over such a scale or yield consistent results. Although the investigation of foraging and dispersal movement over the scale of several kilometers may be infeasible, investigations into the resulting genetic connectivity of populations are likely to be more profitable.

It may be possible to address the biases of the common methods for tracking bumble bees by pairing them with other, more ancillary, methods. For example, because dispersal distances may be difficult to measure, even with genetic mark-recapture, our estimates of dispersal can be paired with studies using captive individuals within tethered flight mills to determine movement capacity (Minter et al. 2018). This complementary approach would help address the problem of limited captures of queen-worker pairs between years (e.g., Lepais et al. 2010, Carvell et al. 2017) and remove the influence of cognitive factors affecting movement, allowing research to focus on the physical capacity under controlled scenarios.

Another common shortfall of most methods is that the observations of individuals tell us little about their habitat preferences or mobility within those landscapes. By exploiting the tendency of animals to increase residency time within habitat of greater suitability, we can examine the relationship between animal movement and land cover (Schultz and Crone 2001, Brown et al. 2017). In bumble bees, this would functionally mean tracking individuals, such as nestsearching queens, either visually or using radar and measuring aspects like turning angle, movement speed, and step length to determine residency time in different potential nesting habitats. In addition to these two complementary methods, there are certainly many more ways to leverage related techniques and behaviors to further examine bumble bee movement, and researchers should look towards the more developed literature on larger organisms to see where we can begin making inroads.

At present, it is difficult to determine when estimated differences in movement reflect methodological bias versus inherent biological differences between species or landscape context because generally more than one of these factors change among studies. For example, there are eight studies that estimate foraging range for Bombus terrestris. Half of them use genetic mark-recapture, the others are evenly divided among alternate methods, but these also occur in different landscape contexts making separation of methods versus context challenging even for this most widely studied species (Supplemental S1). Despite this limitation on our ability to compare methods, there are consistent rank-order differences in movement between a few widely studied species (Figure 1), suggesting that biological differences in movement can be revealed through repeated estimates across landscapes and methodology. If future studies are to investigate drivers of bumble bee movement patterns, consistency between method and study design is needed as the ability to detect differences among treatments may otherwise be obscured by methodological artifacts.

Although we have outlined the utility of several of the established methods, none of the methods is directly tied to a colony or individual's fitness. This disconnect stands as a key gap in our ability to link assessments of movement propensity, rate, direction, or distance to functional implications for populations even though it seems logical that one should follow from the other (e.g., it is difficult to imagine a scenario where all individuals in the colony experience reduced homing ability 
from pesticide exposure without a negative effect on colony performance). For solitary bees, a link can easily be made between reproductive success and distance from resources, such as was shown by Zurbuchen et al. (2010a, 2010b). However, this "simple" solution is unlikely for bumble bees as replication would become difficult with large colonies of foragers compared to the much more manageable placement of many solitary individuals within nesting blocks.

\section{FUTURE DIRECTIONS}

Future applications of the methods described here should explicitly consider the dynamic nature of movement focusing on life stage, ecological context, environmental drivers, and nutritional influences on movement. At present, most studies are of workers, often with static measures of foraging distance. We encourage practitioners to use available methods to investigate differences between individuals, castes, age classes, body sizes, or differing contexts. More recent studies have begun to reveal the ontogeny of forager flight and differences between individuals (Woodgate et al. 2016), as well as colonylevel variability in foraging effort as the resource landscape changes (Hemberger and Gratton 2018, Pope and Jha 2018). Continued efforts are likely to reveal more about the plasticity and flexibility of movement among life stages, allowing us to understand the impacts of habitat fragmentation, agrochemical exposure, changing climate, and resource availability on the movement patterns and population viability of bumble bees. Studies on queen movement are likely to be especially rewarding as radio telemetry (Hagen et al. 2011, Kissling et al. 2014) and genetic markrecapture (Lepais et al. 2010, Carvell et al. 2017) open new opportunities into investigating this poorly resolved portion of bumble bee life history.

The influence of other environmental factors like temperature, agrochemical exposure, parasitoids, and disease on movement pattern or scale is largely unstudied, even though they are ubiquitous aspects of bee ecology. Studies of foragers indicate high levels of micro-parasite infection in various regions (e.g., Schmid-Hempel and Durrer 1991, Plischuk et al. 2009, Li et al. 2012, Gillespie and Adler 2013, Gillespie et al. 2015), and pesticide exposure strongly affects flower handling and spatial foraging behavior (Gill et al. 2012, Samuelson et al. 2016). These factors likely impact foraging success and homing ability and could affect dispersal motivation and distances of queens in fall and spring. An explicit understanding of these factors could be gained from studies controlling for methodological and species-specific differences but varying ecological context.

A further priority for the research tools described here should be an understanding of how internal state, biotic interactions, and colony-level resource demands influence movement motivation and motion capacity. Primary among such internal state variables is nutrition (Woodard and Jha 2017), which could be investigated using homing, radar tracking, or RFID/optical tagging alongside manipulations of diet. In bumble bees, the relationship between nutrition and movement is poorly understood yet likely to profoundly impact the net displacement of all stages of the life cycle — from the timing and extent of foundress emergence and site selection to the foraging of individual workers. Investigations into nutritional states and how they impact flight motivation versus capability could help reveal the causes and consequences of longdistance movements and whether bumble bees thrive from these displacements or merely tolerate them. In general, studies should advance towards the application of these tools under different natural or experimental conditions to help reveal how bumble bee movement changes with differences in context.

\section{CONCLUSIONS}

Although an understanding of bumble bee movement is needed for species conservation and maintenance of pollination services, the 
methodological difficulties of study limit our ability to close key knowledge gaps. Our review reveals that selection of the appropriate method and analytical techniques will often be context and lifestage specific and there is no single best method. Rather, it is important to match the approach to the specific context and details of the questions considered. In practice, one is likely to select the method which balances logistical constraints and the data necessary to address the question of interest.

Overall, technological advances and an increased awareness of the need for well-informed bumble bee movement data for species conservation provide a promising future for addressing key knowledge gaps. We hope our review has provided practitioners and readers with a streamlined guide to understanding the tools available and the ability to contextualize their advantages, limitations, and appropriate application.

\section{ACKNOWLEDGMENTS}

We thank H. Woodard, C. Stuligross, E. E. Crone, and N. Pope for helpful comments on an earlier draft of this manuscript. JMM was supported by NSF Graduate Research Fellowship Award 1049702. NMW and JMM were supported by NSF DEB 1354022.

\section{AUTHOR CONTRIBUTION}

JMM and NMW conceived and developed this review; JMM wrote the initial draft, and NMW participated in the revisions. Both authors read and approved the final manuscript.

\section{COMPLIANCE WITH ETHICAL STANDARDS}

Conflict of interest The authors declare that they have no conflict of interest.

Un examen des méthodes d'étude du mouvement des bourdons

Butiner / dispersion / marquage-recapture / radiorepérage / membre de fratries
Ein Übersichtsartikel zu Methoden für Untersuchungen zum Flugverhalten von Hummeln

Sammelverhalten / Verbreitung / Fang-Wiederfang / Verfolgung mittels Radiowellen / Verwandschaft

\section{REFERENCES}

Aizen, M. A., C. Smith-Ramírez, C. L. Morales, L. Vieli, A. Sáez, R. M. Barahona-Segovia, M. P. Arbetman, J. Montalva, L. A. Garibaldi, D. W. Inouye, and L. D. Harder. 2019. Coordinated species importation policies are needed to reduce serious invasions globally: The case of alien bumblebees in South America. J. Appl. Ecol. 56:100-106

Allen, A. M., and N. J. Singh. 2016. Linking Movement Ecology with Wildlife Management and Conservation. Front. Ecol. Evol. 3

Andrews, K. R., J. M. Good, M. R. Miller, G. Luikart, and P. A. Hohenlohe. 2016. Harnessing the power of RADseq for ecological and evolutionary genomics. Nat. Rev. Genet. 17:81-92

Arbetman, M. P., I. Meeus, C. L. Morales, M. A. Aizen, and G. Smagghe. 2013. Alien parasite hitchhikes to Patagonia on invasive bumblebee. Biol. Invasions 15 :489-494

Bell, K. L., J. Fowler, K. S. Burgess, E. K. Dobbs, D. Gruenewald, B. Lawley, C. Morozumi, and B. J. Brosi. 2017. Applying Pollen DNA Metabarcoding to the Study of Plant-Pollinator Interactions. Appl. Plant Sci. 5 : 1600124

Bell, K. L., N. de Vere, A. Keller, R. T. Richardson, A. Gous, K. S. Burgess, and B. J. Brosi. 2016. Pollen DNA barcoding: current applications and future prospects. Genome $59: 629-640$

Bhattacharya, M., R. B. Primack, and J. Gerwein. 2003. Are roads and railroads barriers to bumblebee movement in a temperate suburban conservation area? Biol. Conserv. 109:37-45.

Bowers, M. A. 1985. Bumble Bee Colonization, Extinction, and Reproduction in Subalpine Meadows in Northeastern Utah. Ecology 66:914.

Boyle, N. K., A. D. Tripodi, S. A. Machtley, J. P. Strange, T. L. Pitts-Singer, and J. R. Hagler. 2018. A Nonlethal Method to Examine Non-Apis Bees for Mark-Capture Research. J. Insect Sci. 18.

Brown, L. M., R. K. Fuda, N. Schtickzelle, H. Coffman, A. Jost, A. Kazberouk, E. Kemper, E. Sass, and E. E. Crone. 2017. Using animal movement behavior to categorize land cover and predict consequences for connectivity and patch residence times. Landsc. Ecol. 32:1657-1670.

Cameron, S. A. 1981. Chemical signals in bumble bee foraging. Behav. Ecol. Sociobiol. 9:257-260. 
Cameron, S. A., H. C. Lim, J. D. Lozier, M. A. Duennes, and R. Thorp. 2016. Test of the invasive pathogen hypothesis of bumble bee decline in North America. Proc. Natl. Acad. Sci. 113:4386-4391.

Cameron, S. A., J. D. Lozier, J. P. Strange, J. B. Koch, N. Cordes, L. F. Solter, and T. L. Griswold. 2011. Patterns of widespread decline in North American bumble bees. Proc. Natl. Acad. Sci. 108:662-667.

Capaldi, E. A., A. D. Smith, J. L. Osborne, S. E. Fahrbach, S. M. Farris, D. R. Reynolds, A. S. Edwards, A. Martin, G. E. Robinson, G. M. Poppy, and J. R. Riley. 2000. Ontogeny of orientation flight in the honeybee revealed by harmonic radar. Nature 403:537-540.

Cartar, R. V. 2004. Resource Tracking by Bumble Bees: Responses to Plant-Level Differences in Quality. Ecology 85:2764-2771.

Carvell, C., A. F. G. Bourke, S. Dreier, S. N. Freeman, S. Hulmes, W. C. Jordan, J. W. Redhead, S. Sumner, J. Wang, and M. S. Heard. 2017. Bumblebee family lineage survival is enhanced in high-quality landscapes. Nature 543:547.

Carvell, C., W. C. Jordan, A. F. G. Bourke, R. Pickles, J. W. Redhead, and M. S. Heard. 2012. Molecular and spatial analyses reveal links between colony-specific foraging distance and landscape-level resource availability in two bumblebee species. Oikos 121:734-742.

Carvell, C., D. B. Roy, S. M. Smart, R. F. Pywell, C. D. Preston, and D. Goulson. 2006. Declines in forage availability for bumblebees at a national scale. Biol. Conserv. 132:481-489.

Chapman, R. E., J. Wang, and A. F. G. Bourke. 2003. Genetic analysis of spatial foraging patterns and resource sharing in bumble bee pollinators. Mol. Ecol. 12:2801-2808.

Cnaani, J., J. D. Thomson, and D. R. Papaj. 2006. Flower Choice and Learning in Foraging Bumblebees: Effects of Variation in Nectar Volume and Concentration. Ethology 112:278-285.

Collett, T. S. 1996. Insect navigation en route to the goal: multiple strategies for the use of landmarks. J. Exp. Biol. 199:227-235.

Comba, L. 1999. Patch use by bumblebees (Hymenoptera Apidae): temperature, wind, flower density and traplining. Ethol. Ecol. Evol. 11:243-264.

Cooke, S. J., S. G. Hinch, M. Wikelski, R. D. Andrews, L. J. Kuchel, T. G. Wolcott, and P. J. Butler. 2004. Biotelemetry: a mechanistic approach to ecology. Trends Ecol. Evol. 19:334-343.

Crall, J. D., N. Gravish, A. M. Mountcastle, and S. A. Combes. 2015. BEEtag: A Low-Cost, Image-Based Tracking System for the Study of Animal Behavior and Locomotion. PLoS One 10:e0136487.

Crall, J. D., C. M. Switzer, R. L. Oppenheimer, A. N. F. Versypt, B. Dey, A. Brown, M. Eyster, C. Guérin, N. E. Pierce, S. A. Combes, and B. L. de Bivort. 2018. Neonicotinoid exposure disrupts bumblebee nest behavior, social networks, and thermoregulation. Science 362:683-686.
Cresswell, J. E., J. L. Osborne, and D. Goulson. 2000. An economic model of the limits to foraging range in central place foragers with numerical solutions for bumblebees. Ecol. Entomol. 25:249-255.

Darvill, B., M. E. Knight, and D. Goulson. 2004. Use of Genetic Markers to Quantify Bumblebee Foraging Range and Nest Density. Oikos 107:471-478.

Darvill, B., S. O’Connor, G. C. Lye, J. Waters, O. Lepais, and D. Goulson. 2010. Cryptic differences in dispersal lead to differential sensitivity to habitat fragmentation in two bumblebee species. Mol. Ecol. 19:53-63.

Dramstad, W. E. 1996. Do bumblebees (Hymenoptera: Apidae) really forage close to their nests? J. Insect Behav. 9:163-182.

Dukas, R., and L. Edelstein-Keshet. 1998. The Spatial Distribution of Colonial Food Provisioners. J. Theor. Biol. 190:121-134.

Elliott, S. E. 2009. Subalpine bumble bee foraging distances and densities in relation to flower availability. Environ. Entomol. 38:748-756.

Fabre, J.-H. 1918. The mason-bees. Dodd, Mead.

Free, J. B. 1993. Insect pollination of crops. 2nd Edition. Academic Press Inc.

Gill, R. J., O. Ramos-Rodriguez, and N. E. Raine. 2012. Combined pesticide exposure severely affects individual- and colony-level traits in bees. Nature 491:105.

Gillespie S. D., and Adler L. S. 2013. Indirect effects on mutualisms: parasitism of bumble bees and pollination service to plants. Ecology 94:454-464.

Gillespie, S. D., K. Carrero, and L. S. Adler. 2015. Relationships between parasitism, bumblebee foraging behaviour, and pollination service to Trifolium pratense flowers. Ecol. Entomol. 40:650-653.

Gosterit, A., and V. C. Baskar. 2016. Impacts of commercialization on the developmental characteristics of native Bombus terrestris (L.) colonies. Insect. Soc. 63:609-614.

Goulson, D. 2009. Bumblebees: Behaviour, Ecology, and Conservation. 2 edition. Oxford University Press, Oxford; New York

Goulson, D., G. C. Lye, and B. Darvill. 2008. Decline and Conservation of Bumble Bees. Annu. Rev. Entomol. 53:191-208.

Goulson, D., E. Nicholls, C. Botias, and E. L. Rotheray. 2015. Bee declines driven by combined stress from parasites, pesticides, and lack of flowers. Science 347:1255957.

Goulson, D., and J. Stout. 2001. Homing ability of the bumblebee Bombus terrestris (Hymenoptera: Apidae). Apidologie 32:105-111.

Greenleaf, S. S., N. M. Williams, R. Winfree, and C. Kremen. 2007. Bee foraging ranges and their relationship to body size. Oecologia 153:589-596.

Grixti, J. C., L. T. Wong, S. A. Cameron, and C. Favret. 2009. Decline of bumble bees (Bombus) in the North American Midwest. Biol. Conserv. 142:75-84. 
Hadfield, J. D., D. S. Richardson, and T. Burke. 2006. Towards unbiased parentage assignment: combining genetic, behavioural and spatial data in a Bayesian framework. Mol. Ecol. 15:3715-3730.

Hagen, M., M. Wikelski, and W. D. Kissling. 2011. Space use of bumblebees (Bombus spp.) revealed by radiotracking. PLoS One 6:e19997.

Hagler, J. R., S. E. Naranjo, S. A. Machtley, and F. Blackmer. 2014. Development of a standardized protein immunomarking protocol for insect mark-capture dispersal research. J. Appl. Entomol. 138:772-782.

Hanski, I. 1998. Metapopulation dynamics. Nature 396:4149.

Harder, L. D. 1986. Influences on the density and dispersion of bumble bee nests (Hymenoptera: Apidae). Ecography 9:99-103.

Haskell, J. P., M. E. Ritchie, and H. Olff. 2002. Fractal geometry predicts varying body size scaling relationships for mammal and bird home ranges. Nature 418:527-530.

Heinrich, B. 1979. "Majoring" and "Minoring" by Foraging Bumblebees, Bombus Vagans: An Experimental Analysis. Ecology 60:246-255.

Hemberger, J., and C. Gratton. 2018. Floral resource pulse decreases bumble bee foraging trip duration in central Wisconsin agroecosystem. Ecol. Entomol. 43.

Holyoak, M., R. Casagrandi, R. Nathan, E. Revilla, and O. Spiegel. 2008. Trends and missing parts in the study of movement ecology. Proc. Natl. Acad. Sci. 105:1906019065.

Ims, R. A., and N. G. Yoccoz. 1997. Studying transfer processes in metapopulations: emigration, migration, and colonization. Pages 247-265 Metapopulation biology. Elsevier.

Jacobson, M. M., E. M. Tucker, M. E. Mathiasson, and S. M. Rehan. 2018. Decline of bumble bees in northeastern North America, with special focus on Bombus terricola. Biol. Conserv. 217:437-445.

Jha, S., and C. Kremen. 2013. Resource diversity and landscape-level homogeneity drive native bee foraging. Proc. Natl. Acad. Sci. 110:555-558.

Jha, S., L. Stefanovich, and C. Kremen. 2013. Bumble bee pollen use and preference across spatial scales in human-altered landscapes. Ecological Entomology 38:570-579.

Jønsson, K. A., A. P. Tøttrup, M. K. Borregaard, S. A. Keith, C. Rahbek, and K. Thorup. 2016. Tracking Animal Dispersal: From Individual Movement to Community Assembly and Global Range Dynamics. Trends Ecol. Evol. 31:204-214.

Kadoya, T., and I. Washitani. 2010. Predicting the rate of range expansion of an invasive alien bumblebee (Bombus terrestris) using a stochastic spatio-temporal model. Biol. Conserv. 143:1228-1235.

Keller, A., N. Danner, G. Grimmer, M. Ankenbrand, K. von der Ohe, W. von der Ohe, S. Rost, S. Härtel, and I. Steffan-Dewenter. 2015. Evaluating multiplexed next- generation sequencing as a method in palynology for mixed pollen samples. Plant Biol. 17:558-566.

Kerr, J. T., A. Pindar, P. Galpern, L. Packer, S. G. Potts, S. M. Roberts, P. Rasmont, O. Schweiger, S. R. Colla, L. L. Richardson, D. L. Wagner, L. F. Gall, D. S. Sikes, and A. Pantoja. 2015. Climate change impacts on bumblebees converge across continents. Science 349:177-180.

Kerr, N. Z., E. E. Crone, and N. M. Williams. 2019. Integrating vital rates explains optimal worker size for resource return by bumblebee workers. Funct. Ecol. $33: 467-478$.

Kissling, W. D., D. E. Pattemore, and M. Hagen. 2014. Challenges and prospects in the telemetry of insects: Insect telemetry. Biol. Rev. 89:511-530.

Klein, A.-M., B. E. Vaissiere, J. H. Cane, I. SteffanDewenter, S. A. Cunningham, C. Kremen, and T. Tscharntke. 2007. Importance of pollinators in changing landscapes for world crops. Proc. R. Soc. B Biol. Sci. 274:303-313.

Kraus, F. B., S. Wolf, and R. F. A. Moritz. 2009. Male flight distance and population substructure in the bumblebee Bombus terrestris . J. Anim. Ecol. 78:247-252.

Kremen, C., N. M. Williams, M. A. Aizen, B. GemmillHerren, G. LeBuhn, R. Minckley, L. Packer, S. G. Potts, T. Roulston, I. Steffan-Dewenter, D. P. Vázquez, R. Winfree, L. Adams, E. E. Crone, S. S. Greenleaf, T. H. Keitt, A.-M. Klein, J. Regetz, and T. H. Ricketts. 2007. Pollination and other ecosystem services produced by mobile organisms: a conceptual framework for the effects of land-use change. Ecol. Lett. 10:299314.

Kremen, C., N. M. Williams, and R. W. Thorp. 2002. Crop pollination from native bees at risk from agricultural intensification. Proc. Natl. Acad. Sci. 99:1681216816.

Leonard, A. S., A. Dornhaus, and D. R. Papaj. 2011. Flowers help bees cope with uncertainty: signal detection and the function of floral complexity. J. Exp. Biol. 214:113-121.

Leonard, A. S., and D. R. Papaj. 2011. 'X' marks the spot: The possible benefits of nectar guides to bees and plants. Funct. Ecol. 25:1293-1301.

Lepais, O., B. Darvill, S. O'Connor, J. L. Osborne, R. A. Sanderson, J. Cussans, L. Goffe, and D. Goulson. 2010. Estimation of bumblebee queen dispersal distances using sibship reconstruction method: Estimation of bumblebee queen dispersal. Mol. Ecol. 19:819-831.

Li, J., W. Chen, J. Wu, W. Peng, J. An, P. Schmid-Hempel, and R. Schmid-Hempel. 2012. Diversity of Nosema associated with bumblebees (Bombus spp.) from China. Int. J. Parasitol. 42:49-61.

Lihoreau, M., N. E. Raine, A. M. Reynolds, R. J. Stelzer, K. S. Lim, A. D. Smith, J. L. Osborne, and L. Chittka. 2012. Radar Tracking and Motion-Sensitive Cameras on Flowers Reveal the Development of Pollinator Multi-Destination Routes over Large Spatial Scales. PLoS Biol. 10:e1001392. 
Long, J. A., and T. A. Nelson. 2013. A review of quantitative methods for movement data. Int. J. Geogr. Inf. Sci. 27:292-318.

Makinson, J. C., J. L. Woodgate, A. Reynolds, E. A. Capaldi, C. J. Perry, and L. Chittka. 2019. Harmonic radar tracking reveals random dispersal pattern of bumblebee ( Bombus terrestris ) queens after hibernation. Sci. Rep. 9:4651.

Malfi, R. L., J. A. Walter, T. H. Roulston, C. Stuligross, S. McIntosh, and L. Bauer. 2018. The influence of conopid flies on bumble bee colony productivity under different food resource conditions. Ecol. Monogr. 88:653-671.

Manning, A. 1956. Some Aspects of the Foraging Behaviour of Bumble-Bees. Behaviour 9:164-200.

Martin, A. P., N. L. Carreck, J. L. Swain, D. Goulson, M. E. Knight, R. J. Hale, R. A. Sanderson, and J. L. Osborne. 2006. A modular system for trapping and massmarking bumblebees: applications for studying food choice and foraging range. Apidologie 37:341-350.

McFrederick, Q. S., and S. M. Rehan. 2016. Characterization of pollen and bacterial community composition in brood provisions of a small carpenter bee. Mol. Ecol. 25:2302-2311.

Menzel, R., U. Greggers, A. Smith, S. Berger, R. Brandt, S. Brunke, G. Bundrock, S. Hülse, T. Plümpe, and F. Schaupp. 2005. Honey bees navigate according to a map-like spatial memory. Proc. Natl. Acad. Sci. U. S. A. 102:3040-3045.

Minter, M., A. Pearson, K. S. Lim, K. Wilson, J. W. Chapman, and C. M. Jones. 2018. The tethered flight technique as a tool for studying life-history strategies associated with migration in insects. Ecol. Entomol. 43:397-411.

Morales, C. L., M. P. Arbetman, S. A. Cameron, and M. A. Aizen. 2013. Rapid ecological replacement of a native bumble bee by invasive species. Front. Ecol. Environ. 11:529-534.

Nathan, R., W. M. Getz, E. Revilla, M. Holyoak, R. Kadmon, D. Saltz, and P. E. Smouse. 2008. A movement ecology paradigm for unifying organismal movement research. Proc. Natl. Acad. Sci. 105:1905219059.

Nichols, J. D., and A. Kaiser. 1999. Quantitative studies of bird movement: a methodological review. Bird Study 46:S289-S298.

van Nieuwstadt, M. G. L., and C. E. R. Iraheta. 1996. Relation between size and foraging range in stingless bees (Apidae, Meliponinae). Apidologie 27:219-228.

Nunes-Silva, P., M. Hrncir, J. T. F. Guimarães, H. Arruda, L. Costa, G. Pessin, J. O. Siqueira, P. de Souza, and V. L. Imperatriz-Fonseca. 2018. Applications of RFID technology on the study of bees. Insect. Soc. 66:15-24

O'Connor, S. A. 2013. The Nesting Ecology of Bumblebees. PhD Thesis, University of Stirling.

O’Connor, S., K. J. Park, and D. Goulson. 2012. Humans versus dogs; a comparison of methods for the detection of bumble bee nests. J. Apic. Res. 51:204-211.
Ogilvie, J. E., and J. D. Thomson. 2016. Site fidelity by bees drives pollination facilitation in sequentially blooming plant species. Ecology 97:1442-1451.

Ollerton, J., R. Winfree, and S. Tarrant. 2011. How many flowering plants are pollinated by animals? Oikos 120:321-326.

Osborne, J. 1., S. j. Clark, R. j. Morris, I. h. Williams, J. r. Riley, A. d. Smith, D. r. Reynolds, and A. s. Edwards. 1999. A landscape-scale study of bumble bee foraging range and constancy, using harmonic radar. J. Appl. Ecol. 36:519-533.

Osborne, J. L., A. P. Martin, N. L. Carreck, J. L. Swain, M. E. Knight, D. Goulson, R. J. Hale, and R. A. Sanderson. 2008. Bumblebee Flight Distances in Relation to the Forage Landscape. J. Anim. Ecol. 77:406415.

Ovaskainen, O., H. Rekola, E. Meyke, and E. Arjas. 2008. Bayesian Methods for Analyzing Movements in Heterogeneous Landscapes from Mark-Recapture Data. Ecology 89:542-554.

Papi, F. 2012. Animal homing. Springer Science \& Business Media.

Pasquet, R. S., A. Peltier, M. B. Hufford, E. Oudin, J. Saulnier, L. Paul, J. T. Knudsen, H. R. Herren, and P. Gepts. 2008. Long-distance pollen flow assessment through evaluation of pollinator foraging range suggests transgene escape distances. Proc. Natl. Acad. Sci. 105(36):13456-13461

Plischuk, S., R. Martín-Hernández, L. Prieto, M. Lucía, C. Botías, A. Meana, A. H. Abrahamovich, C. Lange, and M. Higes. 2009. South American native bumblebees (Hymenoptera: Apidae) infected by Nosema ceranae (Microsporidia), an emerging pathogen of honeybees (Apis mellifera). Environ. Microbiol. Rep. 1:131-135.

Pope, N. S., and S. Jha. 2017. Inferring the foraging ranges of social bees from sibling genotypes sampled across discrete locations. Conserv. Genet. 18:645-658. 1-14.

Pope, N. S., and S. Jha. 2018. Seasonal Food Scarcity Prompts Long-Distance Foraging by a Wild Social Bee. Am. Nat. 191:45-57.

Raine, N. E., and L. Chittka. 2007. The Adaptive Significance of Sensory Bias in a Foraging Context: Floral Colour Preferences in the Bumblebee Bombus terrestris. PLoS One 2:e556.

Raine, N. E., and L. Chittka. 2008. The correlation of learning speed and natural foraging success in bumble-bees. Proc. R. Soc. B Biol. Sci. 275:803-808.

Rao, S., G. Hoffman, J. Kirby, and D. Horne. 2019. Remarkable long-distance returns to a forage patch by artificially displaced wild bumble bees (Hymenoptera: Apidae). J. Apic. Res. 0:1-9.

Rao, S., and J. P. Strange. 2012. Bumble Bee (Hymenoptera: Apidae) Foraging Distance and Colony Density Associated With a Late-Season Mass Flowering Crop. Environ. Entomol. 41:905-915.

Rathcke, B. J., and E. S. Jules. 1993. Habitat fragmentation and plant-pollinator interactions. Curr. Sci. 65:273277. 
Rau, P. 1929. Experimental studies in the homing of carpenter and mining bees. J. Comp. Psychol. $9: 35$.

Redhead, J. W., S. Dreier, A. F. Bourke, M. S. Heard, W. C. Jordan, S. Sumner, J. Wang, and C. Carvell. 2016. Effects of habitat composition and landscape structure on worker foraging distances of five bumblebee species. Ecol. Appl. 26:726-739.

Reynolds, A. M., A. D. Smith, R. Menzel, U. Greggers, D. R. Reynolds, and J. R. Riley. 2007. Displaced Honey Bees Perform Optimal Scale-Free Search Flights. Ecology 88:1955-1961.

Reynolds, D. R., and J. R. Riley. 2002. Remote-sensing, telemetric and computer-based technologies for investigating insect movement: a survey of existing and potential techniques. Comput. Electron. Agric. 35:271-307.

Riley, J. R., U. Greggers, A. D. Smith, D. R. Reynolds, and R. Menzel. 2005. The flight paths of honeybees recruited by the waggle dance. Nature 435:205.

Rossel, S. 1993. Navigation by bees using polarized skylight. Comp. Biochem. Physiol: A: Comparative physiology 104:695-708

Rundlöf, M., G. K. S. Andersson, R. Bommarco, I. Fries, V. Hederström, L. Herbertsson, O. Jonsson, B. K. Klatt, T. R. Pedersen, J. Yourstone, and H. G. Smith. 2015. Seed coating with a neonicotinoid insecticide negatively affects wild bees. Nature.

Samuelson, E. E. W., Z. P. Chen-Wishart, R. J. Gill, and E. Leadbeater. 2016. Effect of acute pesticide exposure on bee spatial working memory using an analogue of the radial-arm maze. Sci. Rep. 6:38957

Schmid-Hempel, P., and S. Durrer. 1991. Parasites, Floral Resources and Reproduction in Natural Populations of Bumblebees. Oikos 62:342-350.

Schmid-Hempel, R., M. Eckhardt, D. Goulson, D. Heinzmann, C. Lange, S. Plischuk, L. R. Escudero, R. Salathé, J. J. Scriven, and P. Schmid-Hempel. 2014. The invasion of southern South America by imported bumblebees and associated parasites. J. Anim. Ecol. 83:823-837.

Schultz, C. B., and E. E. Crone. 2001. Edge-mediated dispersal behavior in a prairie butterfly. Ecology 82:1879-1892.

Silcox, D. E., J. P. Doskocil, C. E. Sorenson, and R. L. Brandenburg. 2011. Radio frequency identification tagging: a novel approach to monitoring surface and subterranean insects. Am. Entomol. 57:86-93.

Sirois-Delisle, C., and J. T. Kerr. 2018. Climate changedriven range losses among bumblebee species are poised to accelerate. Sci. Rep. 8:14464.

Sivakoff, F. S., J. A. Rosenheim, and J. R. Hagler. 2012. Relative dispersal ability of a key agricultural pest and its predators in an annual agroecosystem. Biol. Control 63:296-303.

Southwick, E. E., and S. L. Buchmann. 1995. Effects of Horizon Landmarks on Homing Success in Honey Bees. Am. Nat. 146:748-764.
Sutcliffe, O. L., and C. D. Thomas. 1996. Open Corridors Appear to Facilitate Dispersal by Ringlet Butterflies (Aphantopus hyperantus) between Woodland Clearings. Conserv. Biol. 10:1359-1365.

Suzuki, Y., L. G. Kawaguchi, and Y. Toquenaga. 2007. Estimating nest locations of bumblebee Bombus ardens from flower quality and distribution. Ecol. Res. 22:220-227.

Switzer, C. M., and S. A. Combes. 2016. Bombus impatiens (Hymenoptera: Apidae) display reduced pollen foraging behavior when marked with bee tags vs. paint. J. Melittol. 0:1-13.

Thomson, J. D. 1982. Patterns of Visitation by Animal Pollinators. Oikos 39:241-250.

Thomson, J. D. 1996. Trapline foraging by bumblebees: I. Persistence of flight-path geometry. Behav. Ecol. 7:158-164.

Tomkiewicz, S. M., M. R. Fuller, J. G. Kie, and K. K. Bates. 2010. Global positioning system and associated technologies in animal behaviour and ecological research. Philos. Trans. Royal Soc. London B: Biol. Sci. 365:2163-2176.

Walther-Hellwig, K., and R. Frankl. 2000. Foraging habitats and foraging distances of bumblebees, Bombus spp.(Hym., Apidae), in an agricultural landscape. J. Appl. Entomol. 124:299-306.

Wang, J. 2004. Sibship Reconstruction From Genetic Data With Typing Errors. Genetics 166:1963-1979.

Westphal, C., I. Seffan-Dewenter, and T. Tscharntke. 2006. Foraging trip duration of bumblebees in relation to landscape-wide resource availability. Ecol. Entomol. 31:389-394.

White, G. C., and R. A. Garrott. 2012. Analysis of wildlife radio-tracking data. Elsevier.

Wikelski, M., R. W. Kays, N. J. Kasdin, K. Thorup, J. A. Smith, and G. W. Swenson. 2007. Going wild: what a global small-animal tracking system could do for experimental biologists. J. Exp. Biol. 210:181-186.

Wikelski, M., J. Moxley, A. Eaton-Mordas, M. M. LopezUribe, R. Holland, D. Moskowitz, D. W. Roubik, and R. Kays. 2010. Large-range movements of neotropical orchid bees observed via radio telemetry. PLoS One 5:e10738.

Williams, N. M., E. E. Crone, T. H. Roulston, R. L. Minckley, L. Packer, and S. G. Potts. 2010. Ecological and life-history traits predict bee species responses to environmental disturbances. Biol. Conserv. 143:22802291.

Williams, N. M., J. Regetz, and C. Kremen. 2011. Landscape-scale resources promote colony growth but not reproductive performance of bumble bees. Ecology 93:1049-1058.

Williams, P. H., and J. L. Osborne. 2009. Bumblebee vulnerability and conservation world-wide. Apidologie 40:367-387.

Wolf, S., D. P. McMahon, K. S. Lim, C. D. Pull, S. J. Clark, R. J. Paxton, and J. L. Osborne. 2014. So Near and Yet So Far: Harmonic Radar Reveals Reduced Homing 
Ability of Nosema Infected Honeybees. PLoS One 9:e103989.

Woodard, S. H., and S. Jha. 2017. Wild bee nutritional ecology: predicting pollinator population dynamics, movement, and services from floral resources. Curr. Opin. Insect Sci. 21:83-90.

Woodgate, J. L., J. C. Makinson, K. S. Lim, A. M. Reynolds, and L. Chittka. 2016. Life-Long Radar Tracking of Bumblebees. PLoS One 11:e0160333.

Woodgate, J. L., J. C. Makinson, K. S. Lim, A. M. Reynolds, and L. Chittka. 2017. Continuous Radar Tracking Illustrates the Development of Multi-destination Routes of Bumblebees. Sci. Rep. 7:17323
Zurbuchen, A., S. Cheesman, J. Klaiber, A. Müller, S. Hein, and S. Dorn. 2010a. Long foraging distances impose high costs on offspring production in solitary bees. $\mathrm{J}$. Anim. Ecol. 79:674-681.

Zurbuchen, A., L. Landert, J. Klaiber, A. Müller, S. Hein, and S. Dorn. 2010b. Maximum foraging ranges in solitary bees: only few individuals have the capability to cover long foraging distances. Biol. Conserv. 143:669-676.

Publisher's note Springer Nature remains neutral with regard to jurisdictional claims in published maps and institutional affiliations. 